\title{
Characterization Speckle Effect on Measurement of Blood Flow Using Sensor Based on Self- Mixing Interferometry
}

\author{
Ahmad Zaki Dzulfikar ${ }^{1}$, Agus Rubiyanto ${ }^{1}$, and Endarko ${ }^{1}$
}

\begin{abstract}
The applications of Self-Mixing Interferometry (SMI) have been popular in many fields, including biomedical signals. The self-mixing effect occurs from the coherent backcoupling of the reflected or scattered lights from a target surface. The reflected lights will be detected by a photodiode which has been integrated in one device with the laser. That's why the SMI sensor is quite practical, affordable and simple. However, SMI has the serious problem with the presence of speckle effect in measured signal. The speckle effect produced by the human tissue is called "biospeckles." The biospeckles observed from the skin tissues contain information about the blood flow in dermal capillarities, heartbeat, and others. These biospeckle patterns cause random modulations that will be detected as random amplitude and spectrum by photodiode. In this paper we present a technique to characterize speckle effect on measurement of blood flow in fingertip using sensor based on Self-Mixing Interferometry (SMI). We used a laser diode 785 $\mathrm{nm}$ as a light source and a constant current of $70 \mathrm{~mA}$ as a current source which is irradiated on the skin tissue in the fingertip. Then, the backscattered light reenters the laser cavity and it will be detected by photodiode. The SMI signal with speckle effect will be processed by Continuous Wavelet Transform for reconstruction and detection fringe. Signal processing results show that the number of detected speckle fringes depends largely on determining the number of wavelet waves and the scale used. The fringe pattern resulting from the reconstruction of the signal can be used to determine the frequency of speckles due to object movement. The average speckle frequency of fingertip is $0,5-0,7 \mathrm{~Hz}$.
\end{abstract}

Keywords-Self-mixing Interferometry, Speckle Effect, Continuous Wavelet Transform.

\section{INTRODUCTION}

Over the past several decades, different non-invasive methods for the acquisition of biomedical signals have been developed. The development of non-invasive methods for the acquisition and interpretation of cardiovascular signals, blood flow velocity have gained importance in the last years because of the increase in cardiovascular diseases around the world[1]. A sophisticated method for blood flow velocity measurements is the use of the self-mixing effect in a semiconductor laser diode[2].

Self-mixing interferometry (SMI) method is based on the interaction of a cavity field with the field backscattered

\footnotetext{
${ }^{1}$ Ahmad Zaki Dzulfikar, Agus Rubiyanto, and Endarko are with Department of Physics, Institut Teknologi Sepuluh Nopember, Surabaya, 60111, Indonesia. E-mail: zaki.dzulfikar@gmail.com; bagus.rubi65@gmail.com; cendarko@physics.its.ac.id.
}

from a target. The self-mixing effect occurs from the coherent back-coupling of the reflected or scattered lights from a target surface into the laser cavity where it mixes with the original light of the laser[2]. The main advantage of SMI method is high resolution, low cost, the simple construction[3].

However, SMI method has a s specially relevant issue when it works focused on to diffusive targets[4]. This issue is the presence of speckle effect that caused amplitude modulation of a SMI signal is influenced by the target surface roughness[5].

The speckle effect produced by the human tissue is called "biospeckles." The biospeckles observed from the human skin contain information about the blood flow in dermal capillarities, heartbeat, and other physiological activity[6].

When the laser diode illuminates the skin, the first scattering occur in the skin surface. The multiple absorption and scattering events are caused by the surrounding moving and stationary tissues. Only a small portion of the initial incident light reaches the moving particles, which are mostly the red blood cells, in these capillaries. These particles have irregular shapes and sizes; moreover, they move in random directions and speeds in the capillaries. Therefore, light will be scattered in varying amplitudes in different directions. When the total scattered light is measured at a photodiode, a complex random intensity variation is observed. Summing up the effects of these random multiple scattering events and the roughness of the skin surface, the detected random intensity variations are regarded as biospeckles. These biospeckle patterns cause random modulations occur in the amplitude and spectrum of the laser field. These random modulations show themselves as random intensity variations when detected by the monitor PD inside the laser package behind the rear facet of the $\mathrm{LD}[2]$.

In this thesis focuses on how to characterization speckle effect in the wrist and finger tip. Jukka[7] said that the wrist in the human hand offers a fascinating location for a noninvasive measurement device. The main arteries in the wrist, especially the radial artery, are close to the skin surface and consequently pulsation can be easily detected. In addition, the measurement location is very handy, since the pulsation is easily detected and the measurement device is easy to put on[7]. We will compare the result speckle measurement in fingertip that is commonly used for 
measuring heart beat, concentration of SPO2 and other activity.

\section{METHOD}

The experimental setup is shown in Figure $1 \mathrm{~A}$ laser diode Thorlab L785P090 with nominal wavelength of 785 $\mathrm{nm}$ were used as the light source. The LD is placed on a laser tube that contains a thorlabs LT110P collimator lens and it is connected to a constant current driver LDC202C with $70 \mathrm{~mA}$. The SMI signal is recovered using the laser internal PD which is connected to photodiode driver. The finger and wrist in sequence are fixed on a mechanical fixation which is locked on optical bench so that it can avoid automatic tremble. After preconditioning and filtering, the signal was acquired by a computer via a data acquisition card (arduino uno). Finally, a series of signals processing would be taken using mathematical transform and digital filtering by computer software Origin.

In this research, we demonstrate the use of the Continuous Wavelet Transform (CWT) to process SMI signals in time and frequency domains simultaneously for extracting speckle effect. The accuracy of the method depends upon the precise determination of the position and number of fringes, which are often corrupted by external factors such us white noise, impulsive noise, or speckle. These terms complicate the fringe detection algorithms and may result in measurement errors due to missing fringes.
The use of sliding window to determine the envelope of the SMI signal and locate the correct fringes for target reconstruction[5].

The use of the Morlet CWT enabled to remove white and impulsive noise present in the SMI signal, and to extract target vibration parameters (e.g. amplitude, frequency, velocity). Furthermore, the use of Morlet CWT to recover the envelope of SMI signals proved to be an advantage in those cases where speckle induced signal fading. The method is computationally very eficient as compared to envelope detection to track speckle[4]. After reconstructing signal, we can count the fringe speckle for the determination of the target displacement, although it is not always reliable enough because of different types of noise present in the signal, such as non-linearities at the photo detector, the modulating intensity or the amplification electronics, among other possible causes[8].

\section{RESULT}

After measurement process, the speckle effect affects the detected SMI signal in this experiment. The typical speckle SMI signal is shown in Figure 3 . The surface of the fingertip destroy the spatial coherence of the laser beam, the operation of the LD is affected by the speckle effect[3] The serious speckle effect gives rise to interferometric fringe missing or changing in the SMI interferometer[9].

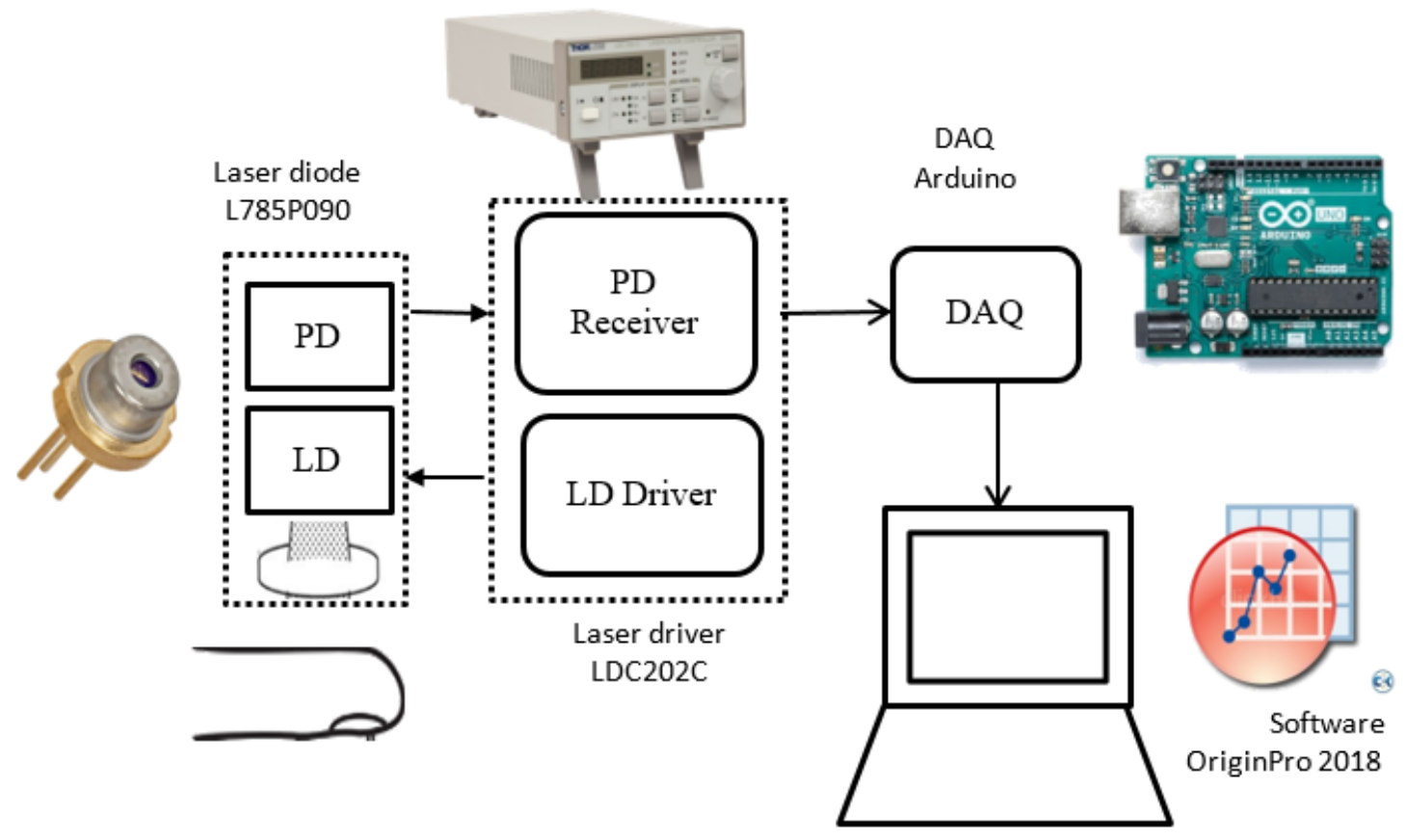

Figure 1. Experimental Setup 


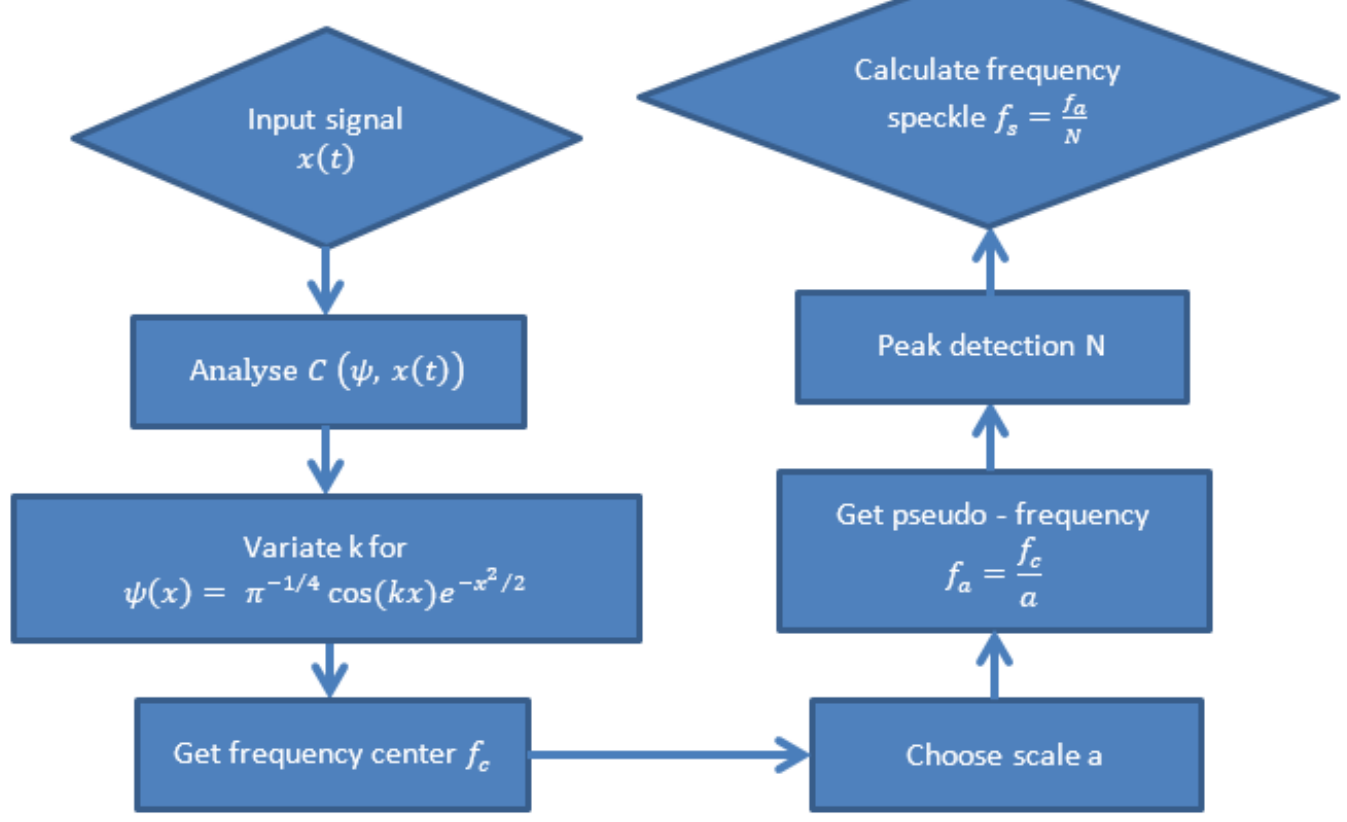

Figure 2. Flow Chart signal processing

(a)

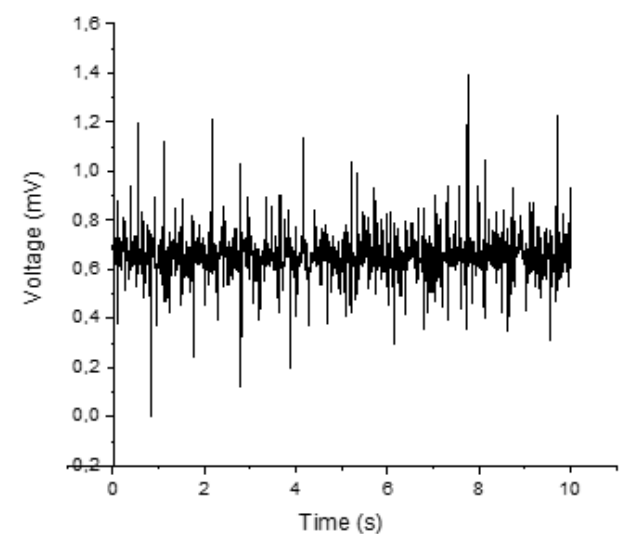

(b)

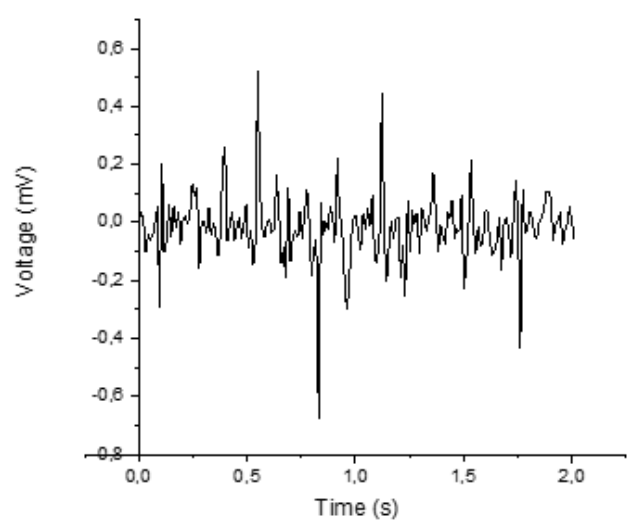

Figure 3. Typical speckle SMI signal (a) Original speckle (b) Zooming speckle

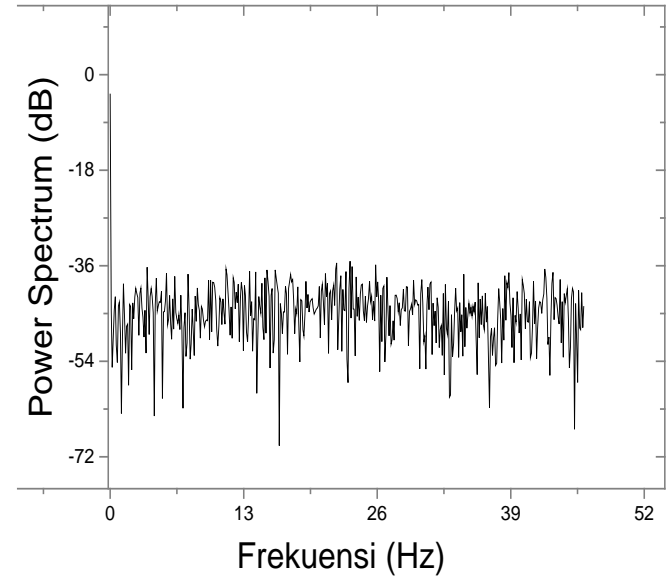

Figure 4. Power spectrum of the detected signal.
The power spectrum of the SMI signal in Figure 4. was also calculated to have information on the frequency components of the signal. A signal dominated by the detection of the Doppler-shifted light field will have a peak value at a certain frequency corresponding to the target surface velocity. In the case of the blood or dye solutions, the Doppler peak will still be present; however, it will be broadened. On the other hand, if the detected signal is dominated by the speckle field, then no such distinct peaks will appear; instead, we will have a broad range of frequencies with no dominant peak

However, the power spectrum of the SMI signal obtained has a broad range of frequencies but does not show a dominant peak. Instead, we see that the amplitude is almost constant (non decaying) value at a specific frequency..

After the SMI signal is processed by CWT, we get the signal reconstruction in Figure 5. This signal not only helps 
to detection fringes due to speckle, but also enables to determine the time instance when the target motion changes its direction. In below, the Table. 1 shows the comparison

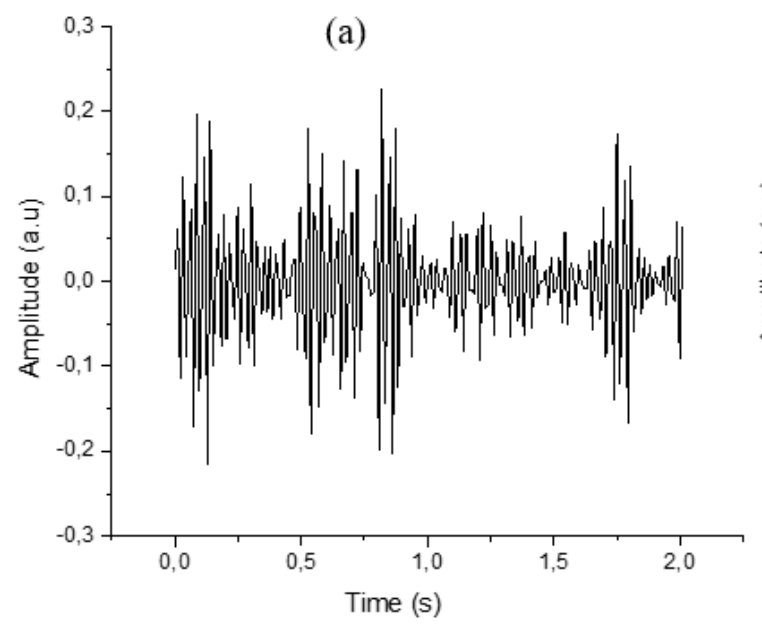

between wave number 10 and 15 with variation of scale a $(4,8,16)$. Using Origin software, we can get pseudo frequency and frequency speckle.

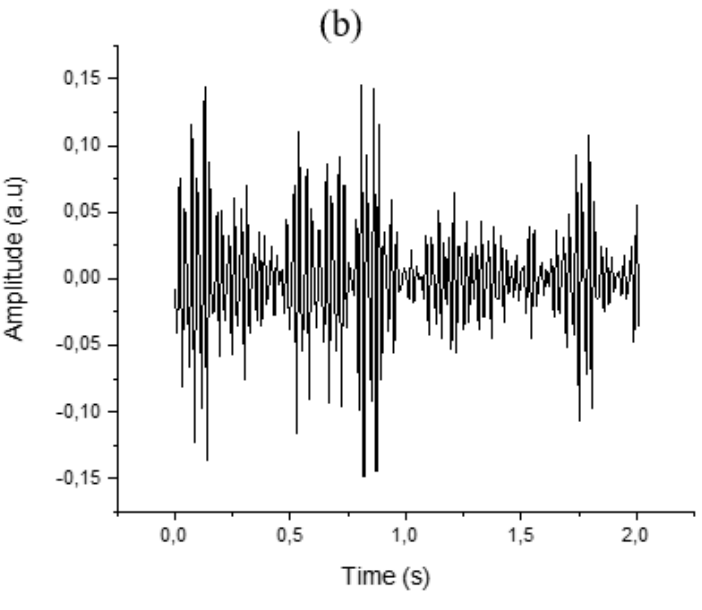

Figure 5. The signal reconstruction (a) for wave number $=10$ and scale $=4$ (b) for wave number $=15$ and scale $=4$

TABLE 1.

THE COMPARISON BETWEEN WAVE NUMBER 10 AND 15

\begin{tabular}{|c|c|c|c|c|c|}
\hline Wave Number & frequency Center $f_{c}(\mathrm{~Hz})$ & Scale a & Pseudo Frequency $f_{a}(\mathrm{~Hz})$ & Peak Speckle N & Frequency SPECKLE $f_{s}(\mathrm{~Hz})$ \\
\hline & & 4 & 36,14739 & 72 & 0,502047083 \\
\hline \multirow[t]{3}{*}{10} & 144,575705 & 8 & 18,07369 & 36 & 0,502046944 \\
\hline & & 16 & 9,03685 & 18 & 0,502047222 \\
\hline & & 4 & 54,94403 & 75 & 0,732587067 \\
\hline \multirow[t]{2}{*}{15} & 294,934438 & 8 & 27,47201 & 54 & 0,508740926 \\
\hline & & 16 & 13,73601 & 28 & 0,490571786 \\
\hline
\end{tabular}

\section{CONCLUSION}

The characterization of SMI speckle with Morlet CWT is very efficient. We can calculate the fringe speckle easily from the reconstructed signal. The number of detected speckle fringes depends largely on determining the number of wavelet waves and the scale used. The fringe pattern resulting from the reconstruction of the signal can be used to determine the frequency of speckles due to object movement. The average speckle frequency of fingertip is 0,5-0,7 Hz.

\section{REFERENCES}

[1] A. Arasanz, F. J. Azcona, S. Royo, A. Jha, and J. Pladellorens, “A new method for the acquisition of arterial pulse wave using selfmixing interferometry,” Opt. Laser Technol., vol. 63, pp. 98-104, Nov. 2014.

[2] S. K. Ozdemir, S. Takamiya, S. Ito, S. Shinohara, and H. Yoshida, "Self-mixing laser speckle velocimeter for blood flow measurement,” IEEE Trans. Instrum. Meas., vol. 49, no. 5, pp. 1029-1035, 2000.

[3] Y. Wei et al., "Double-path acquisition of pulse wave transit time and heartbeat using self-mixing interferometry," Opt. Commun., vol. 393, pp. 178-184, Jun. 2017.
[4] A. Jha, F. J. Azcona, C. Yañez, and S. Royo, "Extraction of vibration parameters from optical feedback interferometry signals using wavelets,” Appl. Opt., vol. 54, no. 34, p. 10106, Dec. 2015.

[5] U. Zabit, O. D. Bernal, and T. Bosch, "Self-mixing laser sensor for large displacements: signal recovery in the presence of speckle," IEEE Sens. J., vol. 13, no. 2, pp. 824-831, Feb. 2013.

[6] S. K. Ozdemir, I. Ohno, and S. Shinohara, "A comparative study for the assessment on blood flow measurement using self-mixing laser speckle interferometer,” IEEE Trans. Instrum. Meas., vol. 57, no. 2, pp. 355-363, 2008.

[7] J. Hast, "Self-mixing interferometry and its applications in noninvasive pulse detection,” University of Oulu, 2003.

[8] A. Jha, S. Royo, F. Azcona, and C. Yanez, "Extracting vibrational parameters from the time-frequency map of a self mixing signal: An approach based on wavelet analysis," in IEEE SENSORS 2014 Proceedings, 2014, pp. 1881-1884.

[9] S. Donati, G. Martini, and T. Tambosso, "Speckle pattern errors in self-mixing interferometry,” IEEE J. Quantum Electron., vol. 49, no. 9, pp. 798-806, Sep. 2013. 